\title{
Using iterated greedy and randomized iterated greedy algorithms to solve urban area waste collection in Riyadh city
}

\author{
Abdulwahab Almutairi * \\ School of Mathematics, Unaizah College of Sciences and arts, Qassim University, Saudi Arabia \\ *Corresponding author E-mail: a.abdulwahab.a@gmail.com
}

\begin{abstract}
This paper addresses the real-life waste collection vehicle routing problem by applying Iterated Greedy (IG) and Randomized Iterated Greedy (RIG) in order to improve the processes. This kind of problem becomes more complex in developing countries in several aspects such as costs and fuel. Nowadays, the waste collection is considered as one of the interesting areas. There are three types of waste: commercial, residential and roll-on-roll-off. In this paper, we mainly consider the residential waste collection problem. The problem can be summarized as follows: a vehicle has to satisfy the demand at each customer location while satisfying the capacity of the vehicle for reducing the total cost. We report a case study that is related to waste collection in Riyadh, Kingdom of Saudi Arabia. To solve the case study problem, IG and RIG were employed. Experiments have been done on the case study data and show a better performance when compared IG algorithm results with RIG algorithm results.
\end{abstract}

Keywords: Case Study; Iterated Greedy; Randomization; Waste Collection

\section{Introduction}

In recent years, the vehicle routing problem (VRP) is considered one of the biggest discussed combinatorial optimization problems. Different types of algorithms techniques have been implemented in order to solve VRP. The VRP can be defined as a creating a number of routes for a number of known vehicles that start from a central depot to serve a number of customers locations and then return back to a central depot, without exceeding the vehicle capacity at minimum total travel cost. Waste collection problems have been receiving increasing attention from many researchers. Also, waste collections have been developed and implemented in different vehicle routing problems. The waste collection has been divided into three areas: commercial, residential, and roll-on-roll-off also each area is very different from each other. The aim of the waste collection problem is to improve the routing of waste collection vehicles and minimizing the total travel costs. The rest of the paper is organized as follows. Section 1.3 presents the literature review. Section 1.4 describes the methodology used to solve the refuse collection system. Section 1.5 presents the computational results of the case study. Finally, Section 1.6 concludes the paper.

\section{Logistics systems in waste collection: Riyadh case description}

\subsection{Riyadh municipality}

Riyadh is the capital city of the Kingdom of Saudi Arabia with a population of about six million. Riyadh municipality covers an area of more than 2000 square kilometres. The annual municipal waste has increased since 2000 . The Riyadh Municipality represented by the General Administration of Hygiene has designed a waste management system in the city based on administrative and geographical divisions. Cleaning contracts are being implemented in Riyadh by company. The concompany try to treat the waste collection by using the recent modern technological advancement. The waste collection produces high operational costs besides other large expenditure such as car petrol. As a result, the main part of the problem is the routing plan of vehicles as it constitutes a large part of the total operational cost. A problem description is given in the following section.

\subsection{Problem description}

The case study is formulated as a vehicle routing problem and is dealing with collecting a set of geographically dispersed customer waste from different locations. This problem represents the solution of 10 contracts and these contracts have almost 100 areas of supervision in Riyadh. The number of customers to be served in the selected areas of study is almost 551 customers. The case study has different type of 
vehicles therefore, the number of the vehicle is 937 . The capacity of the vehicle varies from 16.82 to 3 . There are two shifts and only one depot where all vehicles start and terminate. Every customer location is visited by one vehicle and all waste at the customer is gathered as a whole (nothing is left behind). The waste quantity of each customer is deterministically known and all waste must be collected. The decision maker should consider the driver time service and rest. In the case study, there are two types of hours' services: the first type is inside the neighbourhood and internal street which is once a day from $9.30 \mathrm{pm}$ to $1 \mathrm{am}$. The second type is in the main street which is twice a day from 2 am to 8 am and from $11 \mathrm{am}$ to $7 \mathrm{pm}$. The objective is to minimize the total costs during the collection stage, while determining concurrently the number of vehicles needed to accomplish the services and while satisfying constraints on vehicle capacity and the duration of each daily route. Due to the lack of information, a plan was made to implement the following activities in order to reach the goal of solving the collection problem: data collection, data verification and validation, data processing, problem detection, problem solving and solution implementation. A sample dataset of the waste collection problem in a district located at Riyadh in Saudi Arabia had been selected, which consisted of ten areas with varied number of customers, as displayed in Table 1. It shows the contract code, the number of each contract driver and the total capacity for each contract with different vehicles.

Table 1: Brief Description About the Case Study

\begin{tabular}{|c|c|c|c|c|}
\hline Municipality code & Sub- Municipality & Contract code & Number of drivers & Capacity \\
\hline $\begin{array}{l}38 \\
42\end{array}$ & $\begin{array}{l}\text { Arqa } \\
\text { Al-mather }\end{array}$ & 121 & 62 & 49 \\
\hline 44 & Rawda & 122 & 126 & 101 \\
\hline 45 & Al-Areegeh & 123 & 74 & 59 \\
\hline 34 & Nimar & & & \\
\hline 47 & Al-shfa & 124 & 116 & 93 \\
\hline 49 & Alaaziziyah & 124 & 110 & 93 \\
\hline 50 & Hayer & & & \\
\hline 37 & Alnaseem & 125 & 132 & 106 \\
\hline 39 & Al-Sali & & & 100 \\
\hline 36 & Al-shmeisi & 126 & 71 & 54 \\
\hline 48 & Al-alia & 127 & 83 & 64 \\
\hline 43 & North & 128 & 87 & 69 \\
\hline 35 & Al-batha & 129 & 110 & 88 \\
\hline 41 & Malaz & 130 & 76 & 60 \\
\hline
\end{tabular}

\section{Literature review}

In recent years, waste collection problem is considered as an important logistic activity within any city. The waste collection business is divided into three major areas: commercial, residential and roll-on-roll-off (2). Therefore, several publications in the waste collection problem have used different methodologies and tools in order to solve the waste problem. Also, these methodologies and tools can be used to reduce the operational cost as well as fuel consumption, support economic development and both reduce and eliminate adverse impacts of waste materials on human health and environment. The idea behind the waste collection problem is to start from the central depot to collect waste from several customers and then return to the central depot while taking into consideration the capacity of the vehicle. The target is to minimize the travel cost and provide a good service.

The Vehicle Routing Problem (VRP) is an important area in computational problem. Moreover, it's very useful for solving real world applications. Generally, the VRP is computationally very hard, and cannot be solved by exact methods; therefore, the literature proposes many heuristics such as saving algorithm, sweep algorithm and nearest neighbour. (1). Therefore, a number of studies have reviewed a wide range of studies and methods with different assumptions. Also, a number of solutions have been published in the literature to solve these case studies. Several studies of waste collection are available in the literature. (6) presented a good table that described several benchmarks of studies that had solved real case studies related to waste collection problem.

Many papers are based on different metaheuristic approaches. For example, (4) presents an efficient heuristic with Monte Carlo Simulation in order to improve a case study of urban waste collection in smart cities. They used Kim et al. (2006) benchmarks and the average gap of their results shows the best solution in 4 of the 10 benchmark instances. In 2017, (6) cooperate with Northern part of Peninsular Malaysia waste collection service provider in order to provide a benchmark for the waste collection problem, which is based on real-life problem settings taken from their case studies and includes six areas with different number of customers. Also, they considered one depot and one disposal with 7 tonnes of the vehicle capacity were used to serve up to 103 customers. These benchmarks are used to apply and test the proposed method as presented in the paper. Their results show better solutions (11.09\%) then than actual distance travelled using the present system.

(3) used Geographical Information System (GIS) methodological structure in order to design the time and cost for the waste transportation. This method has been divided into four different parts and each part has a huge amount of cost for waste collection. In terms of the computational results, the have presented a better performance in the fuel consumption and operational costs. (8) used Internet of Things (IOT), sensor and android application in order to make the cities greener and smart. They have a system to measure the volume of the waste and then send the information to the authorities. Therefore, the main advantage of this step is to help the workers to locate the waste bin location of the customer with the help of an android application.

The key contribution of this paper is to compare the method "Randomised IG with IG" to the real life case study problem and to minimise the total cost. The vehicle capacity has been considered as $100 \%$ during the design stage. If the vehicle capacity is exceeded, recourse actions have to be planned to make sure the feasibility of solutions in case of rout failure.

\section{Methodology}

This section proposed the two types of algorithms in this case study, Iterated Greedy algorithm and Randomized Iterated Greedy algorithm. Note that both types of algorithms could be applied to the same instance of the waste collection problem and each algorithm constructs one or more vehicle routes in such a way that each route is terminated whenever the capacity of the vehicle constraint is violated. Full capacity of the vehicle has been considered during the designing of the routes. In the iterated greedy algorithm, the sequence of solutions is generated by using two phases: destruction and construction while in the randomized iterated greedy algorithm, the process of the iterated greedy 
algorithm is extended by adding the randomization in order to improve the initial solution for the first phase of the algorithm. The details of each algorithm are explained next.

This work was performed by employing a modified version of the IG algorithm introduced by (10) in order to solve the waste collection problem. The IG algorithm is conceptually simple and works by generating a sequence of solutions by iterating over a greedy construction heuristic using two central procedures consisting of the destruction and the construction phases. In the destruction phase, a number of solution customers are randomly removed from a previously constructed complete candidate solution. In the next step, the construction procedure is applied, in which the customers removed in the destruction phase are inserted into the solution until a complete candidate solution is obtained. An acceptance criterion is applied once a candidate solution has been completed to decide whether the new solution will replace the incumbent solution. The process of IG is then iterated over these steps until a stopping criterion is met, such as a computation time limit or a maximum number of iterations or the minimum solution is found.

In terms of randomization, the probability can be assigned to the destruction phase then continue to implement the IG algorithm approach. The above explanation of the IG algorithm is when the demand is deterministic and the safety stocks in all vehicles are not considered. The solutions for the deterministic case were computed using the IG algorithm. After that randomization was added by assigning different probabilities to each potential customer. The customers with high probability are then be chosen first, before customers with low probability, although all customers are selected in the end. It is also important to note that in doing so, an attempt is made to avoid the issue of selecting the appropriate size of the restricted list, and to provide a guarantee that the probabilities of being selected are always proportional to the position of each customer in the route. The procedure of IG algorithm approach presented earlier can be extended to solve the waste collection problem.

\section{Computational results}

The main performance measure used is the capacity of the vehicle. Besides this, we reported additional measures during the planning horizon: minimize the number of vehicles and utilization for the loading capacity. Therefore, these measures lead to a reduction the total costs. This section show the heuristics applied to the real case study and report the obtained results. In this result, a comparison was done for the IG and RIG. The two algorithms proposed in this paper are coded using Java application. A Macintosh HD on $2.3 \mathrm{GHz}$ Intel HD Graphics 40001024 MB with Intel Core i7 with 4 GB 1600 MHz DDR3 has been used to perform the computational experiments. Table 2 shows the results achieved for real case study by using both Iterated Greedy (IG) and Randomized IG (RIG). The proposed heuristic is tested and verified using the benchmarks provided by the company. The project has 10 contracts and each contract cover a specific area and the results over all contracts can be seen in Table 2. The results for a particular instance are shown in the following columns. In the first column, there are 10 contracts in the case study that has been considered. The second column represents how many areas can each contract cover which is called the supervisory area. The number of routes that are used to solve the problem is shown in the third column. The fourth and the fifth columns represent the total costs that have been achieved by using both methods. Moreover, the percentage deviation formula between the approaches is calculated as follows;

Percentage deviation $=\left(\frac{(I G)-(R I G)}{(R I G)}\right) * 100$.

Table 2: Summary Table of 10 Contracts Investigated in Part Riyadh Municipality

\begin{tabular}{|c|c|c|c|c|c|c|}
\hline Problem sets & Supervisory area & Routes & Total costs (1) (IG) & Total costs (2) (RIG) & GAP (1)-(2) & Waste generated in $(\mathrm{kg})$ \\
\hline Contract code_1 & 8_areas & 98 & 1583.5 & 1533.5 & $3.26 \%$ & 1637.78 \\
\hline Contract code_2 & $1 \overline{3} \_$areas & 150 & 1742.3 & 1695.3 & $2.77 \%$ & 2596.95 \\
\hline Contract code_3 & 7_areas & 90 & 1454.3 & 1428.3 & $1.82 \%$ & 1509.78 \\
\hline Contract code_4 & $1 \overline{4} \_$areas & 158 & 2013 & 1989 & $1.21 \%$ & 3233.36 \\
\hline Contract code_5 & 17_areas & 164 & 2073.5 & 1999.5 & $3.70 \%$ & 2727.69 \\
\hline Contract code_6 & 6_areas & 66 & 1426.5 & 1379.5 & $3.41 \%$ & 897.68 \\
\hline Contract code_7 & 9_areas & 94 & 1485.2 & 1437.2 & $3.34 \%$ & 1774.6 \\
\hline Contract code_8 & $10 \_$areas & 88 & 1337.5 & 1325.5 & $0.91 \%$ & 1625.5 \\
\hline Contract code_9 & 9_areas & 142 & 1752.6 & 1715.6 & $2.16 \%$ & 2114.45 \\
\hline Contract code_10 & 7_areas & 93 & 1501.3 & 1499.3 & $0.13 \%$ & 1496.73 \\
\hline Average & ----------- & ------- & 1636.97 & 1600.27 & $2.27 \%$ & \\
\hline
\end{tabular}

The gaps are expressed as a percentage value between two approaches and the respective gaps between total costs are shown in Table 2 in the sixth column. The last column showed the waste generated for each contract. In Table 2, we summaries the results obtained by the methodologies where the vehicle capacity is considered during the design stage as $100 \%$. The best result for each instance is shown in bold. Notice that Iterated Greedy clearly outperforms the Randomized Iterated Greedy (average gap about 2.27\%).

\section{Conclusion}

In this paper, we have discussed a real-life case study that is related to waste collection in the Capital of Saudi Arabia (Riyadh). The case study is considered as a vehicle routing problem that arises when several known customers' wastes needs to be collected by different vehicles having varying capacity. Empty vehicles leave the central depot and then collect waste from different customers, emptying themselves at waste disposal facilities. Furthermore, the vehicles maybe need to make multiple visits to collect the waste. The main objective is to minimize the total costs of the problem. In the case study, we considered different constraints that relate to real-world considerations. More specifically we took into consideration vehicle capacity associated with customers waste. We also took into consideration the weight of the waste. To solve the problem, an initial solution is applied by assigning each customer to a vehicle using CWS and it was improved by applying the IG and RIG method. Computational tests were done on the case data which showed the improvement of the results between these methods. For further research, the time window should be considered in order to improve the work and reduce the cost. To do this, more efforts are required to gather this kind of data. 


\section{References}

[1] Faccio, M., Persona, A., Zanin, Giorgia. (2011). Waste collection multi objective model with real time traceability data. Waste management. Volume 31, issue 12, December 2011, pages 2391-2405. https://doi.org/10.1016/j.wasman.2011.07.005.

[2] Golden. B. L., Assad., A. A. Wasil. E. A., (2011). Routing vehicles in the real world: applications in the solid waste, beverage, food, dairy, and newspaper industries. Published in the vehicle routing problem, pages 245-286. Society for industrial and applied mathematics Philadelphia, PA, USA, 2011. ISBN: 0-89871-498-2. https://doi.org/10.1137/1.9780898718515.ch10.

[3] Gupte, S, K, Bhatia, R, K., Routing optimization of municipal solid waste collection in Jabalpur city using ARC GIS. (2017). International journal of trend in scientific research and development. ISSN No: 2456-6470. Volume 2. Issue 1. November- December 2017. Page 457. https://doi.org/10.31142/ijtsrd7000.

[4] Gruler, A., Juan, A. A., Fikar, C., Hirsch, P. (2015). A Simheuristic for the Waste Collection Problem with Stochastic Demands in Smart Cities. Simulation in Production and and Logistics. (2015). Markus Rabe \& Uwe Clausen (eds.) Fraunhofer IRB Verlag, Stuttgart 2015.

[5] Kim, J, D., Choi, H., Lee, D., (2007). Vehicle routing in a refuse collection system: a case study. Fifth international conference on computational science and applications. https://doi.org/10.1109/ICCSA.2007.12.

[6] Mat, N, A., Benjamin, A, M., Abdul-Rahman, S., and Wibowo, A, (2017). Nearest greedy for solving the waste collection vehicle routing problem: A case study. AIP conference proceedings 1905, 040018 (2017). https://doi.org/10.1063/1.5012206.

[7] Moustafa, A., Abdulhalim, A, A., Eltawil, A, B., Fors. N., (2013). Waste collection vehicle routing problem: case study in Alexandria, Egypt. 19th International conference on industrial engineering and engineering management. https://doi.org/10.1007/978-3-642-37270-4_89.

[8] Rajan, L., Saji, K, S., Saraswathi, U., (2018). Solid waste management using internet of things and android application. GRD Journal/ Global research and development journal for engineering/ national conference on emerging trends in electrical, electronics and computer engineering (ETEEC-2018) April 2018. E-ISSN: 2455-5703.

[9] Ruiz, R., Stutzle, T., (2005). An Iterated Greedy Algorithm for the Flowshop Problem with Sequence Dependent Setup Times. The 6th Metaheuristics International Conference, 817-823.

[10] Ruiz, R., Stutzle, T. (2007). A simple and effective iterated greedy algorithm for the permutation flowshop scheduling problem. European Journal of Operational Research, 177, 2033-2049. https://doi.org/10.1016/j.ejor.2005.12.009. 